\title{
Coletas de dados por meio de tablets - prático, barato e de fácil programação
}

\section{Data collection using tablets - practical, low-cost and with easy programming}

\section{AUTORES \\ Inaian Pignatti Teixeira ${ }^{1}$ (D) \\ Priscila Missaki Nakamura ${ }^{2,3}$ (D) \\ Bruno de Paula Caraça Smirmaul ${ }^{4}$ (D) \\ Leonardo de Campos ${ }^{3}$ (D) \\ Eduardo Kokubun ${ }^{3}$ \\ 1 Universidade de São Paulo - Escola de \\ Engenharia de São Carlos, São Carlos, São Paulo, Brasil. \\ 2 Instituto Federal de Educacão, Ciência e \\ Tecnologia, Muzambinho, Minas Gerais, Brasil. \\ 3 Universidade Estadual Paulista - Programa de Pós-Graduação em Ciências da Motricidade, Rio Claro, São Paulo, Brasil. \\ 4 Escola Superior de Tecnologia e Educação, Rio Claro, São Paulo, Brasil.}

\section{CONTATO}

Inaian Pignatti Teixeira

inaianteixeira@botmail.com

Av. Trab. São-Carlense, 400, Parque Arnold

Schimidt, São Carlos, São Paulo, Brasil.

CEP: 13566-590.

DOI

10.12820/rbafs.23e0014

\begin{abstract}
RESUMO
Objetivou-se relatar uma experiência de utilização de um dispositivo móvel para coleta de dados, além de descrever os procedimentos para sua implementação. A pesquisa foi composta por dois momentos: entrevistas de 2008 realizadas por meio de papel e caneta e em 2014-2015 por meio de dispositivos móveis. O processo de implementação dos questionários nesses dispositivos móveis seguiu três etapas: i) escolha do dispositivo móvel; ii) escolha do aplicativo; iii) desenvolvimento e programação do aplicativo. Quando comparada à coleta utilizando papel e caneta, a utilização de tablet apresentou uma economia monetária de $25,8 \%$, sendo que, para uma coleta futura, essa economia seria de $99,2 \%$, e uma economia de tempo superior à $56,9 \%$. Os resultados apontam que o uso de tablet para coleta de dados em pesquisas epidemiológicas deve ser encorajado, uma vez que apresenta benefícios espaciais, econômicos e temporais quando comparado ao uso do papel e caneta.
\end{abstract}

Palavras-chave: Coleta de dados; Tecnologia; Epidemiologia.

\section{ABSTRACT}

The study aim was to report an experience of using a mobile device to collect data from an epidemiological survey, and to describe the step-by-step procedures for its implementation. The research consisted of two phases: interviews using paper and pen in 2008 and performed by mobile devices in 2014-2015. The implementation process of the questionnaires on these mobile devices followed three stages: i) choice of mobile device; ii) App choice; iii) development and app programming. When compared to the collection using pen and paper, the use of tablet presented a monetary economy of $25.8 \%$, and, for a future collection, this economy would be $99.2 \%$ and a saving of time greater than $56.9 \%$. The results indicate that the tablet use for data collection in epidemiological research should be encouraged, once it presents spatial, economic and temporal benefits when compared to paper and pen use.

Keywords: Data collection; Technology; Epidemiology.

\section{Introdução}

A pesquisa científica, embasada por procedimentos racionais e sistemáticos, busca por soluções/reflexões sobre um problema ${ }^{1}$. No campo de pesquisa em atividade física e saúde e, mais especificamente na área de epidemiologia, é comum um grande volume de dados a serem coletados, devido à complexidade do processo saúde-doença, exigindo a investigação de vários parâmetros em amos- tras de grande dimensão ${ }^{2}$. Diante disso, o processo de coleta, tabulação e tratamento dos dados consome boa parte do tempo total despendido na pesquisa.

Tradicionalmente, a coleta de dados na área de epidemiologia no Brasil é realizada por meio de questionários, entrevistas ou observações que são registradas por meio de papel e caneta ${ }^{3,4}$ e, posteriormente, ocorre a tabulação dos dados em computadores. 
Uma alternativa à utilização do papel, capaz de facilitar a coleta e tabulação dos dados, é a utilização de dispositivos móveis como netbooks, smartphones, Personal Digital Assistants (PDAs) e tablets ${ }^{5,6}$. Estes dispositivos proporcionam uma série de vantagens: possibilidade de fácil correção de informações, detecção de inconsistências no momento da coleta, inserção de "pulos de questões" de forma automática, além de economia de tempo e dinheiro na tabulação dos dados. Apesar de todos os benefícios, o uso de dispositivos móveis para coletas de campo ainda não se encontra consolidado, principalmente na América Latina, em grande parte pela dificuldade de "programação" do aplicativo no aparelho e gestão da base de dados.

Assim, os objetivos do presente estudo são: relatar uma experiência de utilização de um dispositivo móvel para coleta de dados de um estudo epidemiológico; descrever passo-a-passo os procedimentos para implementação da coleta de dados e realizar uma comparação com os resultados do uso de papel e caneta.

\section{Métodos}

Em 2008, o Núcleo de Atividade Física, Esporte e Saúde e o Laboratório de Atividade Física e Envelhecimento iniciaram uma pesquisa intitulada "Prevalência e fatores associados à inatividade física em adultos do município de Rio Claro, São Paulo (SP)”, contando com a participação de 1.588 adultos $^{7,8}$. O questionário utilizado foi impresso em papel do tamanho A4, sendo composto por 228 perguntas dispostas em 22 folhas.

Em 2014-2015, a segunda etapa da pesquisa foi realizada, acrescentando-se mais 69 questões ao questionário original de 2008. As novas questões foram referentes a utilização da bicicleta em ciclovia/ciclofaixas $(\mathrm{n}=27)$, preferência e tolerância da intensidade de exercícios físicos $(\mathrm{n}=16)$ e uso de academias ao ar livre $(\mathrm{n}=26)$. Porém, com o intuito de facilitar e agilizar a coleta de dados, a segunda etapa do estudo foi realizada por meio de dispositivos móveis. As etapas do processo de implementação dos questionários nesses dispositivos móveis são descritas abaixo:

Para a escolha do dispositivo móvel (hardware), os seguintes critérios foram utilizados: custo do dispositivo, praticidade de manejo, durabilidade da bateria, risco de exposição à acidentes e roubos, e disponibilidade de aplicativos para coleta e gerenciamento de dados.

O levantamento dos possíveis aplicativos (software), cuja função principal seria a coleta e gerenciamento de bases de dados, foi realizado na web e no Google
Play (base específica da plataforma Android). Após o levantamento dos principais aplicativos disponíveis, analisou-se o custo, procedimentos de instalação, facilidade de programação/desenvolvimento da interface de coleta, interatividade com a "nuvem" (armazenamento remoto) e gerenciamento do banco de dados.

Uma vez escolhido o hardware e o software, os processos de programação foram realizados de acordo com as orientações dos fabricantes (ver seção baixo: "Desenvolvimento e programação do ODK”).

\section{Resultados}

Os resultados são apresentados em quatro seções específicas: escolha do hardware; escolha do software; desenvolvimento e programação dos dispositivos e; relato de experiência.

Dentre as principais opções de dispositivos móveis disponíveis no mercado em 2014, foram avaliadas três opções, sendo elas, netbook, smartphone e tablet. Avaliando-se os custos de aquisição do dispositivo, o tablet e o smartphone apresentaram valores iniciais cerca de cinco vezes menores quando comparado ao netbook (Tabela 1).

Outro ponto avaliado foi o tamanho do dispositivo, que influencia a praticidade do manejo e risco de exposição. Nesse sentido, dispositivos muito pequenos tem sua exposição reduzida (diminuindo os riscos de roubos, por exemplo) e sua manipulação é mais fácil (por exemplo: possibilidade de segurar com apenas uma das mãos). Dentre as três opções, o netbook apresentou as maiores dimensões, em função de presença de telas maiores (entre 10 e 13,9 polegadas) e da presença do teclado físico. Outro ponto negativo do netbook é que sua tela, na maior parte dos modelos, não era responsiva ao toque, sendo necessário a utilização do touchpad ou um mouse externo, dificultando ainda mais o manejo do mesmo durante a coleta de dados. Além de não possuírem teclados físicos, as telas de tablets e smartphones mediam entre 7 e 10 polegadas, e menos que 7 polegadas, respectivamente. Nesse sentido, a tela de 7 polegadas dos tablets, ligeiramente maior que o smartphone, apresentou uma melhor dimensão para visualização de fotos, figuras e eventuais vídeos.

Com relação ao peso, o netbook apresentou um peso de aproximadamente $1,2 \mathrm{~kg}$ (para dispositivos com 10 polegadas), enquanto smartphones e tablets eram cerca de 8,0 e 3,75 vezes mais leves, respectivamente (Tabela 1). Adicionalmente, a duração da bateria de um tablet era cerca de $38 \%$ maior que a do smartphone, garantin- 
do a autonomia de um dia de coletas sem a necessidade de recarga do dispositivo. Em função dos critérios supracitados, optou-se pela utilização de tablet de 7 polegadas, com tela responsiva ao toque.

Tabela 1 - Comparativo de custo e características físicas de tablet, smatphone e netbook (Pesquisa de preços realizada em 28/02/2014).

\begin{tabular}{lccc}
\hline & Tablet & Smartphone & Netbook \\
\hline Custo (valor mínimo aproximado)* & $\mathrm{R} \$ 179,00$ & $\mathrm{R} \$ 179,00$ & $\mathrm{R} \$ 979,02$ \\
Tamanho da tela (polegadas) & 7 a 10 & $<7$ & 10 a 13,9 \\
Tela responsiva ao toque & sim & sim & sim\# \\
Peso médio $(\mathrm{kg})$ & 0,32 & 0,15 & 1,2 \\
Carga média da bateria $(\mathrm{mAh})$ & 3600 & 2600 & $>4000$ \\
\hline
\end{tabular}

* www.buscapé.com; \# Apesar de haver modelos com tela responsiva, a grande maioria não apresenta esse recurso.

Uma vez definido o hardware, o próximo passo foi realizar um levantamento e definir o software a ser empregado. Dentre os inúmeros aplicativos encontrados, destacamos os três mais populares e melhores avaliados entre os usuários do Google Play: Epi Info, doForms e o Open Data Kit (ODK).

Os três aplicativos supracitados realizavam coletas sem a necessidade de conexão de dados via internet no momento da coleta, não sendo necessário a tecnologia $3 \mathrm{~g} / 4 \mathrm{~g}$. Em função disso, os dados poderiam ser armazenados no próprio dispositivo e transferidos para a base de dados na "nuvem" assim que o dispositivo se conectasse à uma rede wi-fi. Além dessa vantagem, os três aplicativos eram de fácil programação e com interfaces bem intuitivas.

Com relação aos custos, tanto o Epi Info quanto o ODK eram gratuitos, enquanto o doForms cobrava uma tarifa de 9,95 dólares por mês por tablet, dificultando seu uso para coletas com grande número de entrevistadores ou pesquisas com restrições orçamentárias.

Com relação à instalação, o Epi Info demonstrou ser instável, com erros que resultavam em travamentos, fechamento automático e necessidade de uma versão mais moderna do Android (4.0 ou superior). Por fim, o ODK mostrou-se ser um aplicativo de fácil programação, estável, isento de custos e aceito por todas versões do Android. Diante dos aspectos supracitados, optou-se pela utilização do aplicativo ODK.

O aplicativo ODK oferecia suporte para geolocalização, imagens, faixas de áudio, faixas de vídeo e códigos de barras, bem como as respostas numéricas e textuais. Além disso, era possível a programação de pulos automáticos em função de uma resposta prévia, configuração de modos de exibição diferenciados (em forma de lista, de matriz ou separadas), além da possibilidade de definir a obrigatoriedade de resposta.

O procedimento de desenvolvimento e programação foi dividido em três etapas:

\section{- Programação do formulário}

Para a programação dos formulários, o aplicativo ODK disponibilizava duas opções: ou por meio de sites específicos (ex: enketo.org) ou uma programação manual realizada com auxílio do software Excel. No caso do website Enketo, o plano básico (um formulário por mês) consistia em mensalidades de 6,00 dólares. Dessa forma, optamos pela programação manual utilizando o software Excel. O ODK disponibilizava um modelo para iniciar a programação (https://docs.opendatakit. org/xlsform/) e um detalhado manual de programação (http://xlsform.org/). Basicamente, o arquivo do Excel era composto por duas abas (planilhas), sendo uma destinada para as perguntas e suas características, e outra aba destinada às opções de respostas.

A aba referente às perguntas ("survey") possuia 19 colunas que são preenchidas com as características das perguntas, sendo que apenas as 3 primeiras eram de caráter obrigatório. As 19 possíveis características das perguntas, bem como uma breve descrição e um exemplo, são apresentados no Quadro 1.

Uma vez finalizado a elaboração deste arquivo do Excel, o mesmo deveria ser convertido para o formato XML, cuja principal característica é criar uma infraestrutura única para diversas linguagens de fórmulas.

- Criação da base de dados na "nuvem"

Após o formulário ser testado e aprovado, foi necessário criar a "nuvem" para agregar os dados coletados pelos mesmos. Para isso foi necessário, com auxílio de um computador, seguir sete passos bem descritos no próprio site (https://goo.g1/IIxDiL). Uma vez criada a "nuvem", foi necessário entrar no endereço da aplicação e fazer o upload do arquivo XML gerado na etapa anterior.

\section{- Implantação do questionário no tablet}

Após as etapas anteriores, foi preciso configurar o ODK instalado no tablet com o endereço criado (ex.: https://nome_colocado_na aplicaşão_no_app_engine.appspot.com) de forma a conectar o tablet com a nuvem.

\section{Relato de experiência}

Com relação ao tempo de aplicação do questionário, o tempo médio, na pesquisa utilizando-se de papel e ca- 
Quadro 1 - Características das perguntas a serem utilizadas durante a programação do formulário.

\begin{tabular}{|c|c|c|c|}
\hline \multicolumn{2}{|c|}{ Características das perguntas } & \multirow{2}{*}{ Breve descrição } & \multirow{2}{*}{ Exemplo } \\
\hline Inglês & Português & & \\
\hline Type & Tipo da variável & $\begin{array}{l}\text { Nesse campo é possível selecionar a } \\
\text { natureza da resposta. }\end{array}$ & $\begin{array}{l}\text { Texto, número inteiro, número com casa decimal, } \\
\text { seleção de uma resposta, seleção de múltiplas } \\
\text { respostas, notas, capturar coordenada geográfica } \\
\text { de um ponto, traçar uma rota, traçar um polígono, } \\
\text { por meio de várias coordenadas geográficas, data, } \\
\text { hora, data e hora, capturar imagem, capturar } \\
\text { áudio, capturar vídeo, ler código de barra, realizar } \\
\text { um cálculo e selecionar botão de "confirmar". }\end{array}$ \\
\hline Name & Nome/código & $\begin{array}{c}\text { Nome que dará título à coluna quando } \\
\text { a base de dados for exportada para o } \\
\text { Excel }\end{array}$ & Idade \\
\hline Label & Rótulo & Forma que a pergunta aparecerá no tablet & Qual sua idade? \\
\hline Hint & Dica & $\begin{array}{l}\text { Aparecerá, em letras menores e na cor cinza } \\
\text { claro, logo abaixo do rótulo, uma dical } \\
\text { orientação de como preencher o campo. }\end{array}$ & Anotar idade em anos \\
\hline Constraint & Restrição de resposta & $\begin{array}{l}\text { Estabelece critérios, não permitindo } \\
\text { valores absurdos, digitados errados ou } \\
\text { fora dos critérios estabelecidos. }\end{array}$ & $\begin{array}{l}\text { Só será permitido a entrada de dados (idade) cujo } \\
\text { valor seja igual ou maior que } 60 \text { anos. }\end{array}$ \\
\hline Constraint_message & Mensagem de erro & $\begin{array}{l}\text { Mensagem informando o porquê a } \\
\text { resposta não foi aceita }\end{array}$ & $\begin{array}{l}\text { Considerando os critérios da pesquisa, só serão } \\
\text { aceitos participantes com idade maior ou igual a } \\
60 \text { anos. }\end{array}$ \\
\hline Required & Reposta obrigatória & $\begin{array}{c}\text { O programa só avança ou salva se a } \\
\text { questão assinalada como obrigatória for } \\
\text { respondida }\end{array}$ & O campo de idade é obrigatório. \\
\hline Appearance & Aparência & $\begin{array}{l}\text { As perguntas podem aparecer em forma } \\
\text { separada (uma questão de cada vez na } \\
\text { tela do tablet), em forma de lista ou } \\
\text { tabela (várias de cada vez na mesma tela) }\end{array}$ & $\begin{array}{l}\text { Todas as questões de um determinado bloco do } \\
\text { questionário aparecerão na mesma página em } \\
\text { formato de lista. }\end{array}$ \\
\hline Default & Resposta padrão & $\begin{array}{l}\text { Ao preencher esse campo, o próprio } \\
\text { programa já insere uma resposta padrão. } \\
\text { Assim, o entrevistador só precisará } \\
\text { alterar/preencher caso a resposta seja } \\
\text { diferente da pré-definida. Esse campo } \\
\text { normalmente é utilizado quando a } \\
\text { prevalência de determinada resposta é } \\
\text { imensamente maior que outra. }\end{array}$ & $\begin{array}{c}\text { Algum médico ou profissional da saúde já lhe } \\
\text { disse que o senhor(a) possui Síndrome de Reye? } \\
\text { Reposta padrão=não }\end{array}$ \\
\hline Relevant & Aplicabilidade & $\begin{array}{l}\text { Nesse campo podem ser definidas } \\
\text { condições para aparição da questão. }\end{array}$ & $\begin{array}{l}\text { As perguntas sobre atividade física no domínio } \\
\text { do trabalho só aparecerão para a pessoa que } \\
\text { responder, em uma pergunta prévia, que trabalha } \\
\text { de forma remunerada ou voluntária. }\end{array}$ \\
\hline Read_only & Apenas leitura & $\begin{array}{l}\text { Apenas leitura, não exigindo uma } \\
\text { reposta. Normalmente utilizado para } \\
\text { nomear sessões específicas ou para } \\
\text { apresentar orientações gerais aos } \\
\text { entrevistados. }\end{array}$ & $\begin{array}{c}\text { As próximas perguntar serão relativas a como o } \\
\text { senhor se transporta de um lugar para o outro. } \\
\text { Sendo assim, gostaria que o senhor pensasse } \\
\text { apenas nas atividades físicas específicas nesse } \\
\text { domínio. }\end{array}$ \\
\hline Calculation & Cálculo & $\begin{array}{l}\text { Nesse campo é possível elaborar um } \\
\text { cálculo a ser executado }\end{array}$ & $\begin{array}{l}\text { No campo IMC quero que o ODK faça a } \\
\text { conversão automática (peso*altura }{ }^{2} \text { ) }\end{array}$ \\
\hline Hint::Chinese & dica::chinês & Dica em outro idioma & Chinês \\
\hline Label::Chinese & rótulo:: chinês & Rótulo em outro idioma & Chinês \\
\hline Image & imagem & $\begin{array}{c}\text { Nesse campo pode ser inserida uma } \\
\text { imagem que aparecerá na tela do } \\
\text { dispositivo }\end{array}$ & $\begin{array}{c}\text { Imagem da Escala de Percepção Subjetiva de } \\
\text { Esforço }\end{array}$ \\
\hline Audio & áudio & $\begin{array}{l}\text { Similar à imagem, porém ao invés de } \\
\text { uma imagem, é disponibilizado um } \\
\text { áudio no formato Wav }\end{array}$ & $\begin{array}{l}\text { Áudio de uma respiração durante um exercício } \\
\text { de intensidade vigorosa e de um exercício de } \\
\text { intensidade moderada. }\end{array}$ \\
\hline Image:: Chinese & $\begin{array}{l}\text { Imagem em outro } \\
\text { idioma }\end{array}$ & $\begin{array}{c}\text { Nesse campo pode ser inserida uma } \\
\text { imagem em outro idioma, aparecendo } \\
\text { na tela do dispositivo }\end{array}$ & $\begin{array}{l}\text { Imagem da Escala de Percepção Subjetiva de } \\
\text { Esforço com descriçãao em chinês }\end{array}$ \\
\hline
\end{tabular}


... continua

\begin{tabular}{|cccc|}
\hline Características das perguntas & Português & Breve descrição & Exemplo \\
\hline Inglês & Áudio em outro idioma & $\begin{array}{c}\text { Nesse campo pode ser inserido um } \\
\text { áudio em outro idioma }\end{array}$ & Chinês \\
\hline Media::audio:::Chinese & $\begin{array}{c}\text { Nesse campo pode ser inserido } \\
\text { um vídeo, no formato de mp4, que } \\
\text { aparecerá na tela do dispositivo }\end{array}$ & $\begin{array}{c}\text { Vídeo de uma pessoa executando um exercício de } \\
\text { maneira correta e outro de maneira errada. Após } \\
\text { assistir ambos os vídeos o entrevistado julgará } \\
\text { qual é o certo. }\end{array}$ \\
Media::vídeo & mídia:vídeo &
\end{tabular}

neta (em 2008), foi de aproximadamente 60 minutos $^{7}$, enquanto a coleta com o auxílio dos tablets (em 20142015) foi $38,2 \%$ menor (37,1 \pm 15 minutos). Ao relativizar o tempo gasto pela quantidade de questões contidas nos questionários em cada ano, essa economia de tempo é ainda maior, passando de 22,6 segundos/questão para 9,8 segundos/questão (redução de 56,9\%). Vale destacar que o ganho real de tempo é ainda maior se considerarmos que na coleta por meio de tablets não é necessário a tabulação dos dados como nos questionários de papel.

Considerando que, em 2008, cada questionário continha 22 folhas, ao todo foram utilizadas 34.936 folhas. Assim, o gasto total, ajustado pela inflação-IPCA, foi de $\mathrm{R} \$$ 5.240,21, sendo $\mathrm{R} \$ 4.719,23$ na impressão dos questionários, $\mathrm{R} \$ 415,63$ em pastas para arquivamento, $\mathrm{R} \$ 67,51$ em pranchetas de madeira e $\mathrm{R} \$ 37,84 \mathrm{em} \mathrm{ca-}$ netas esferográficas. Além desses gastos, foram contratados dois digitadores por um período de dois meses, totalizando um valor de $\mathrm{R} \$ 1.125,22$. Por fim, o valor investido nos materiais para coleta e tabulação desses questionários foi de $\mathrm{R} \$ 6.365,43$. Já para o segundo momento da pesquisa, em 2014-2015, foram comprados seis tablets da marca Samsung modelo Galaxy Tab 3, no valor de $R \$ 609,66$ cada, e mais seis capas de proteção por $R \$ 30,00$ cada, totalizando $R \$ 3.837,96$. $\mathrm{O}$ consumo de energia estimando, considerando que cada tablet passasse 8 horas por dia ao longo de um ano de coleta, seria de 29,2 KW por tablet. Transformando em reais (Tarifa Elektro $2015=\mathrm{R} \$ 0,27426 / \mathrm{KW}$ ), isso representaria um gasto de $\mathrm{R} \$ 48,05$ para os seis tablets.

Baseado nas informações acima, a coleta utilizando tablets representou uma economia monetária de 25,84\% $(\mathrm{R} \$ 1.354,20)$. Caso seja realizada uma futura etapa dessa pesquisa (ou similar), o valor a ser gasto seria imensamente menor, uma vez que não seria necessária a aquisição dos tablets. Sendo assim, a projeção de custos com materiais de coleta passaria de $\mathrm{R} \$$ 6.365,43 em 2008 para $R \$ 3.886,01$ em 2014-2015 e, por fim, para $R \$$ 48,05 em uma futura coleta $(0,75 \%$ do valor gasto em 2008). Além disso, vale ressaltar que esses tablets podem ser utilizados em inúmeras outras coletas e pesquisas, representando, dessa forma, uma economia ainda maior.

Além dos benefícios espaciais, econômicos e temporais, outra grande vantagem observada foi a facilidade gerada pelos "pulos" automáticos, conduzindo de forma mais rápida a entrevista, uma vez que não era necessário, baseado na resposta anterior, procurar qual era a próxima pergunta.

Apesar dos inúmeros benefícios apresentados nas sessões anteriores, é necessária uma reflexão quanto aos aspectos de manutenção e segurança na utilização de tablets. Acreditamos que a utilização dos tablets demandará, em algum momento, algum tipo de reparo ou manutenção, porém, esses custos certamente serão mínimos comparados à economia gerada. A utilização de tablet, pode aumentar o risco de roubo do equipamento, entretanto, em caso de um possível roubo, é possível localiza-lo por meio de aplicativos (por exemplo: lostdroid) e bloqueá-lo remotamente. Outra opção seria a aquisição de um seguro para o tablet, que usualmente incluem corbertura contra acidentes, problemas elétricos e roubo.

Outro aspecto a ser considerado é a segurança dos dados, que deve ser analisada sob duas perspectivas: 1 ) enquanto os dados ainda estão no tablet e 2) no processo de transferência e armazenamento na nuvem. Para a primeira situação o tablet, possui inúmeros aplicativos, cuja função é exigir uma senha para iniciar determinado aplicativo. Assim, é possível solicitar uma senha para abrir o ODK, tornando-o acessível apenas por pessoas autorizadas. Com relação ao processo de envio dos dados para a nuvem, o mesmo se dá de forma criptografada usando um certificado SSL de 256 bits, evitando assim possíveis interceptações e garantindo que todos os dados transmitidos sejam sigilosos e seguros. Em nosso caso, uma vez transmitidos, esses dados ficam retidos no servidor do ODK e a única forma de acesso aos mesmo é com posse do nome do usuário e a senha.

Em virtude dos dados analisados e da experiência relatada, podemos concluir que o uso de tablets para coleta de dados apresenta diversas vantagens em subs- 
tituição ao papel e caneta. Assim, esperamos ter contribuído com subsídios para que futuras pesquisas possam optar pela utilização de tal instrumento, principalmente frente à sua facilidade de programação, economia de tempo e dinheiro.

\section{Conflito de interesses}

Os autores declaram não haver conflito de interesses.

\section{Financiamento}

Fundação de Amparo à Pesquisa do Estado de São Paulo (FAPESP [Processo:2012/18795-7]).

\section{Contribuição dos autores}

Teixeira IP, participou da concepção inicial do estudo, redação e revisão crítica do texto, busca da literatura e coleta de dados. Nakamura PM, participou da concepção inicial do estudo, redação e revisão crítica do texto. Smirmaul BPC, participou da concepção inicial do estudo, redação e revisão crítica do texto, busca da literatura e coleta de dados. Campos L, participou da concepção inicial do estudo, redação e revisão crítica do texto e busca da literatura. Kokubun E, participou da concepção inicial do estudo, redação e revisão crítica do texto.

\section{Referências}

1. Andrade MM. Introdução à metodologia do trabalho científico. 10. ed. São Paulo: Atlas, 2010.
2. Khoury MJ, Lam TK, Ioannidis JP, Hartge P, Spitz MR, Buring JE, et al. Transforming epidemiology for 21st century medicine and public health. Cancer Epidemiol Biomarkers Prev. 2013; 22(4):508-16.

3. Barros AJD, Santos IS, Victora CG, Albernaz EP, Domingues MR, Timm IK, et al. Coorte de nascimentos de Pelotas, 2004: metodologia e descrição. Rev Saúde Pública. 2006;40(3):402-13.

4. Duncan BB, Vigo A, Hernandez E, Luft VC, Ahlert H, Bermann K, et al. Gerência de Informação em Estudos Multicêntricos: o Estudo Longitudinal de Saude do Adulto. Rev. Saúde Pública. 2013;47(Supl 2):95-104.

5. Lane SJ, Heddle NM, Arnold E, Walker I. A review of randomized controlled trials comparing the effectiveness of hand held computers with paper methods for data collection. BMC Med Inform Decis Mak. 2006; 6:23.

6. Wilcox AB, Gallagher KD, Boden-Abdala B, Bakken SR. Research data collection methods: from paper to tablet computers. Med Care. 2012;50(7):S68-S73.

7. Nakamura PM, Teixeira IP, Papini CB, Fernandes RA, Kokubun E. Associação da caminhada no lazer e no transporte com ambiente construído em adultos do município de Rio Claro-SP. Rev Bras Ativ Fís Saúde. 2013;18(4):424-34.

8. Nakamura PM, Teixeira IP, Smirmaul BP, Sebastião E, Papini CB, Gobbi S, et al. Health related quality of life is differently associated with leisure-time physical activity intensities according to gender: a cross-sectional approach. Health Qual Life Outcomes. 2014;12:98.

Recebido: 18/12/2017

Aprovado: 08/06/2018

\section{Como citar este artigo:}

Teixeira IP, Nakamura PM, Smirnaul BP, Campos L, Kokubun E. Coletas de dados por meio de tablets - prático, barato e de fácil programação. Rev Bras Ati Fis Saúde. 2018;23:e0014. DOI: 10.12820/rbafs.23e0014 